Volume 8, No.6, November - December 2019

International Journal of Advanced Trends in Computer Science and Engineering

Available Online at http://www.warse.org/IJATCSE/static/pdf/file/ijatcse38862019.pdf

https://doi.org/10.30534/ijatcse/2019/38862019

\title{
Performance Evaluation of Wide Area Network using Cisco Packet Tracer
}

\author{
Sanam. Nagendram ${ }^{1}$, P. Sai Anil ${ }^{1}$, E. V. S. Pavan ${ }^{1}$, V. Amarendra ${ }^{1}$ \\ ${ }^{1}$ Department of Electronics and Communication Engineering, Koneru Lakshmaiah Education Foundation, \\ Vaddeswaram, AP, India.
}

\begin{abstract}
Computer networks have turn out to be most significant in the current society. The huge number of MNC'S hang on the good operative of their networks for communications, management, administration, online business solutions, etc. The WAN is the most necessary computer network used to join the different cities and states So in this paper, with the use of Cisco Packet Tracer we will design a WAN network. This paper defines how we can use the cisco packet simulation tool to improve a simulation model for the WAN network to the cities. The study provides understanding to the different concepts for example designing the topology for WAN networks, the assignment of ip addresses to devices,switches and routers, and the transmission of information using Virtual Wide Area Networks. (VWANs).
\end{abstract}

Key words: VWAN, Cisco packet tracer, IP Addressing, Topology design

\section{INTRODUCTION}

We can create a WAN by connecting large numbers of local area networks. Computer networks were used to share information between different systems. The WAN is an interconnecting network between cities and capitals.. The WAN is the interconnecting network of cities and capitals. At present day the network is accessible from everywhere at any time, so we have to provide the fast and secure connection irrespective of location to every user. The Cisco packet tracer provides the path to calculate the effect of a hardware upgrade, changing topology, and increase of network traffic, or using of latest applications on the network. A WAN network is developed with the use of Cisco Packet Tracer in this document. The paper describes how to use the Cisco packet method to build a WAN simulation system for a state's capital cities. This study delivers awareness into multiple concepts such as the configuration of IP addresses and how to transmit information to a single network in the form of a packet and the use of Virtual Wide Area Networks .CCNA: Cisco Certified Network Associate CCNA [1][3] is a networking certification made by Cisco Systems. It is used by Cisco to identify basic capabilities for the setting up and maintenance of medium-sized networks. This software allows us to connect different computers like routers, switches and different users to establish communication between each other to exchange data. It is also scalable to construct a methodological and reliable .network. Portability is one of the features of the WAN function software.

\section{1) Router Address \\ 2) DNS Address \\ 3) Class Identifier}

Dynamic Host Configuration Protocol (DHCP):

Dynamic Host Configuration Protocol [2][4] is useful to model the host's specific IP address. It is used to provide different types. Subnet mask used to determine whether or not the host is on the network. The sub net mask is used in.

The IP address should be different when we find a network where we should use IP addresses for systems or PCs. Total system is useless if it does not offer unique Ip address. For example, if these two systems don't have different IP addresses, take two systems PC1, PC2, the data coming through it doesn't understand going to a specific system and the systems get corrupted. As we took two computers, we assign Ip addresses manually, according to our example. $\mathrm{s}$ we took two pc's, we assign Ip addresses manually. But in case where there are many pc's or devices it is difficult to assign, so DHCP is used.

\section{USER INTERFACE}

The monitor will be shown in the city's digital topology.

- The Cisco Packet Tracer [5][7] network emulator is easy to implement and provides a clear appeal to the graphical user interface.

- Five switches will be shown, separate VLANs and 1 router will be connected to the VWAN.

- The interface of the command line (CLI) will allow you to adjust it or customize it

\section{Hardware interfaces}

We need certain basic requirements for the usage of cisco packet simulator, which are:-

1.A system with minimum RAM of $512 \mathrm{MB}$

2.a system contain storage of $500 \mathrm{MB}$ minimum disk space

Software interfaces :

The requirements for the project are: 
1. The system with operating system of windows 7 or above

2. The system with the installation of cisco network simulation tool.

\section{Cisco Packet Tracer}

Cisco Packet Tracer[1][2] has two modes namely Physical mode and Logical mode. This Packet Tracer is a developed by the Cisco for virtual design of network architectures and the topologies this tool allows all the users to create their own network designs and simulate according to present world computer networks. This software permits all users to use a graphical command line interface to run the setup of Cisco routers [3] and devices. It is a drag - drop interface which includes to configure IP address of routers, switches, $\mathrm{PC}$ and many more. In this we can configure switches in two different ways. Firstly, by writing some commands in CLI which is Command Line Interface and the other way is by giving IP address to the external devices which are connected for the switch. As Packet Tracer is flexible it allows users to add or it allow user to remove virtual network devices.

\section{IPv4 ADDRESSING}

An IP address is an address given to every system to identify each system during communication and it is in numeric form., Every device is assigned by an its own individual IPp address for communication. An ipv4 address is in size of 32 bits of data, This IP address is derived into different classes based on the requirements, it is a logical address given to users to establish network communication and $2^{32}$ address can be given to users. It is in the form of 132.148.20.100 in which each number is of 8 bits. The ip address is of two various types one of it is network address and also called as service address and another is receiver address. The IP address is designed to establish communication between server and the host and to provide the address to the users to establish the secure communication

\section{Classes of Addresses}

There are classified into five various classes and are shown below

Table 1: Types of IPv4 addressing mode

\begin{tabular}{|l|c|}
\hline Address class & Range \\
\hline Class A Address & $1-127$ \\
\hline Class B Address & $128-191$ \\
\hline Class C Address & $192-223$ \\
\hline Class D Address & $224-239$ \\
\hline Class E Address & $240-255$ \\
\hline
\end{tabular}

\section{Subnet Mask}

The subnet mask is used to identify the subnet allocated to the ip address. The ip address are divided as two parts one is reciever address and another is server address. The subnet perorms bitwise AND operations to identify the network address of ip address[21].

Table 2: Default subnet masks for all network classes

\begin{tabular}{|l|l|l|}
\hline Class & Format & $\begin{array}{l}\text { Default } \\
\text { subnet mask }\end{array}$ \\
\hline A & Network.host.host.host & 255.0 .0 .0 \\
\hline B & Network.host.host.host & 255.255.0.0 \\
\hline C & Network.host.host.host & 255.255.255.0 \\
\hline
\end{tabular}

\section{BASIC WAN ARCHITECTURE}

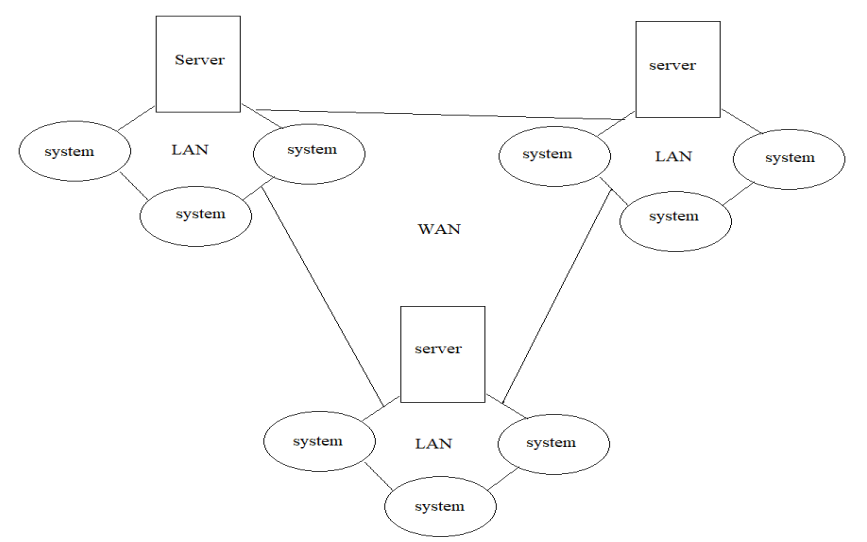

Figure 1: Basic WAN architecture

Wide area network connects multiple number of LANS from different places and forms into a single network. The switches and routers and all the devices combine together and establish the connection between themselves and forms the local area network and a various number of LANs connect each other and forms a single network called WAN network[22].while local network establish connection between colleges and institutes these WAN area networks connects different LANs and establish the communication between different cities. 


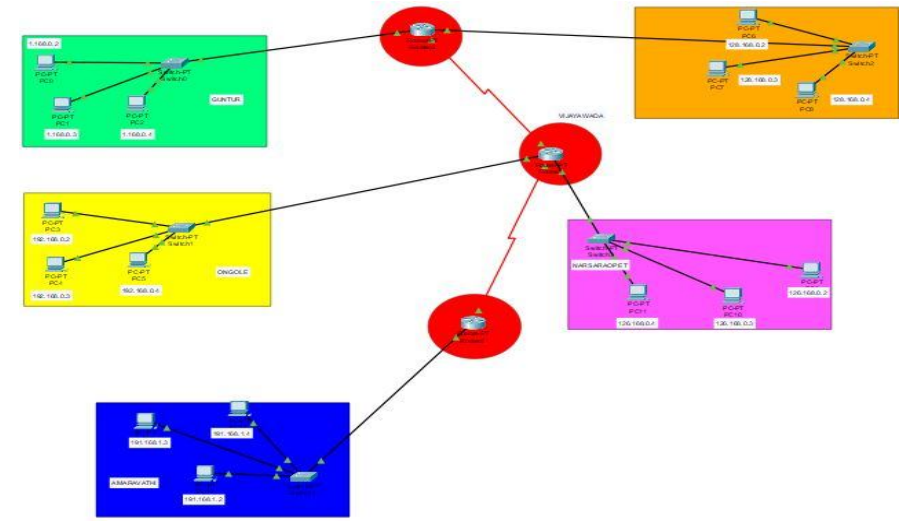

Figure 2: Implemented WAN architecture in Cisco Packet Tracer.

\section{CONFIGURATION}

\section{Router Configuration}

The IP address should be given to router in config option, as see in the diagram Those configurations should be considered according to the interface. Router to Router connection is another impactful thing which should be taken into consideration and in serial ports we can configure the connection to the routers as In the diagram

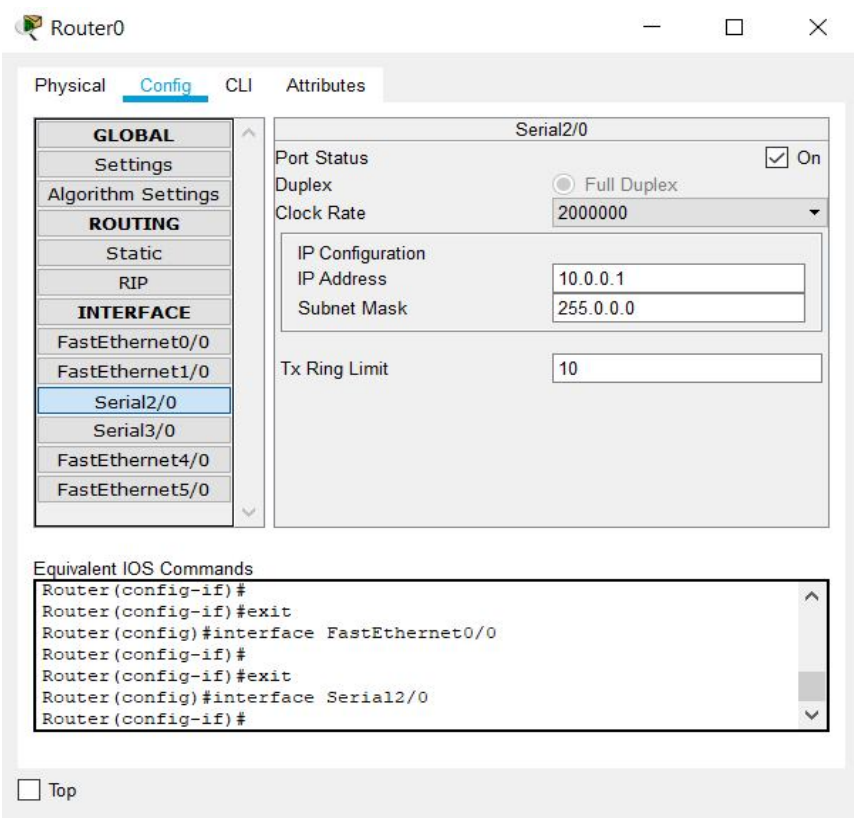

Figure 3: Router configuration

\section{System Configuration}

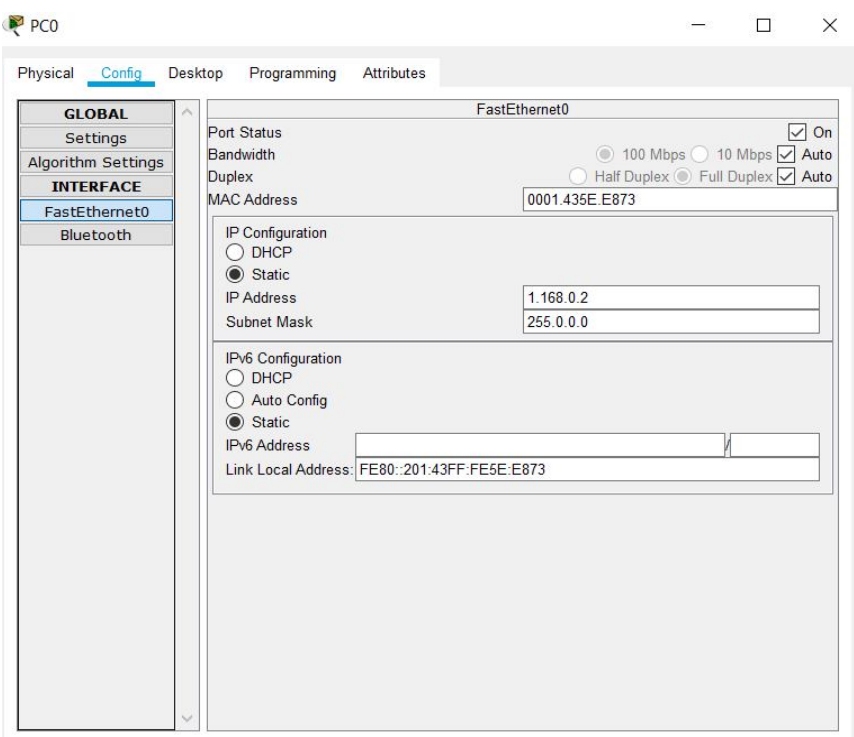

$\sqcap$ Top

Figure 4: System configuration

In system configuration the ip address is assigned to the system and and the router address is filled in the gateway address to establish the connection between the router and the system.

\section{RESULTS}

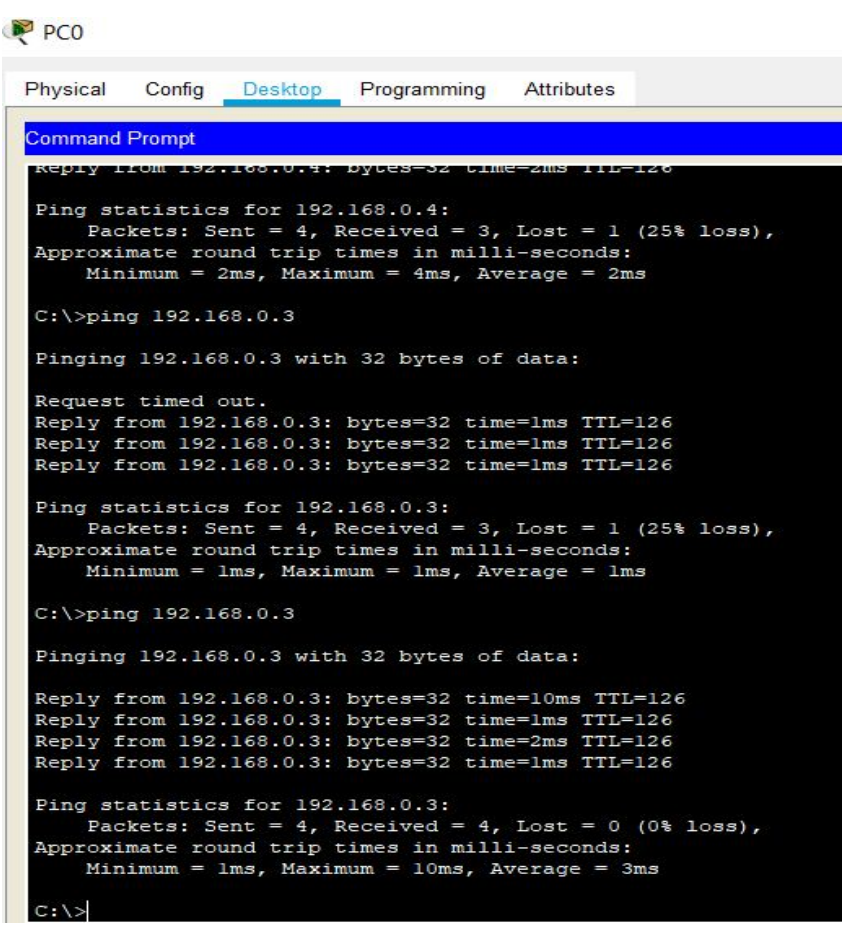




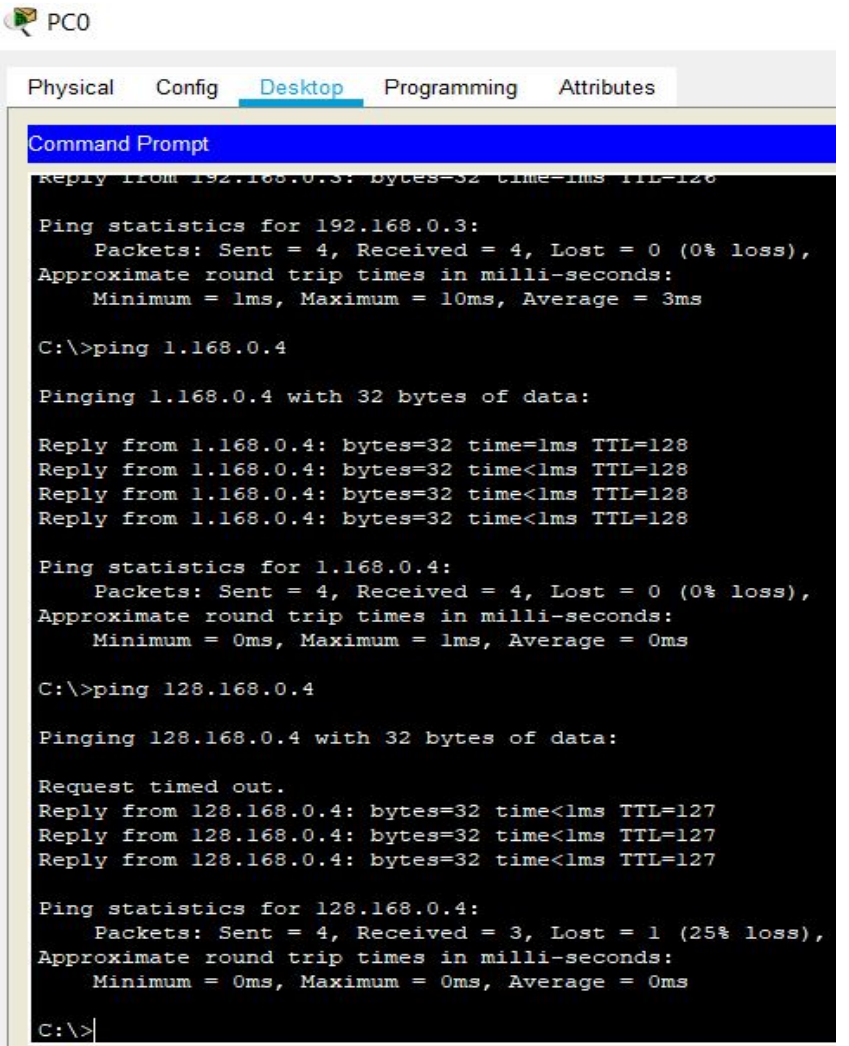

\section{CONCLUSION}

Designing the Wide Area Network scenario provides the substructure for all other system exposures, such as network security and provides the basis for safety, organizational effectiveness, e-learning environments and secure transactions. This paper tells us the designing of network made by Cisco tool, as well as we can be able to implement these scenarios in a district's various locations. In the wide area network, we can also provide email security and further improve its security during message transmission.

\section{References :}

[1] S. Nagendram, K. Ramchand H Rao, " Hybrid Security and Energy Aware Routing for Wireless Ad hoc Networks," International Journal of Recent Technology and Engineering (IJRTE) ISSN: 22773878, Volume-8 Issue-2, July 2019 https://doi.org/10.35940/ijrte.B3659.078219

[2] Massner et.al, "A Dynamic Host Configuration Protocol Based Service Discovery Mechanism," Computer Software and Applications Conference Workshops (COMPSACW), 2011 IEEE 35th Annual, vol., no., pp.62,67, 18-22 July 2011 https://doi.org/10.1109/COMPSACW.2011.21
[3] Data Communications\& Networking (sie) McGraw-Hill Forouzan networking series. Author, A. Behrouz Forouzan et.al .

[4] S. Nagendram, K. Ramchand H Rao " Survey of Different Security and Routing Protocols Hierarchy in Wireless Network,"Communication, International Journal of Engineering and Advanced Technology (IJEAT) ISSN: 2249 - 8958, Volume8, Issue-2S, December 2018

[5] Detection of Invalid Routing Announcements in the RIP Protocol", D. Pei et.al IEEE Global Communications Conference (Globecom), December, 2003

[6] CCNA by Todd Lammle, 5th edition

[7] Todd Lammle et.al Cisco Certified network associate study guide (Wiley Publishing Inc., 2007

[8] Tim Reardon, Planning, Designing and operating local area networks, DISAM Journal, Summer, 1997.

[9] C.R. Lin et.al Adaptive clustering for mobile wireless networks, IEEE Journal on Selected Areas in Communications 15(7)(1997)1265-1275.

https://doi.org/10.1109/49.622910

[10] S.Hedetniemi et.al ,A Survey of gossiping and road casting in communication networks, Networks 18 (4)(1988)319-349.

https://doi.org/10.1002/net.3230180406

[11] Network et.al International Journal of Advanced Research in Computer Science and Software Engineering Volume 3,Issue 2, February 2013.

[12] K. Ramesh Reddy et.al Reactive, Proactive MANET Routing Protocol Comparison. International of Video \&Image Processing and Network Security IJVIPNS-IJENS Vol: 12 No: 05.

[13] Z. Yu et.al A robust group-based key management scheme for wireless sensor networks. In Wireless Communications and Networking Conference, pages 1915-1920. IEEE, 2005.

[14] K. Kifayat et.al. Security in wireless sensor networks. In Handbook of Information and Communication Security, pages 513-552. 2010. https://doi.org/10.1007/978-3-642-04117-4_26

[15] D. Ganesan et al., Highly resilient, energy efficient multipath routing in wireless sensor networks, Mobile Computing and Communications Review 5 (4) (2002) 11-25.

https://doi.org/10.1145/509506.509514

[16] L.M. Feeney, et.al Investigating the energy consumption of an IEEE 802.11 network interface, Technical report T1999-11, Swedish Institute of Computer Science, Kista,Sweden (1999).

[17] OLIVIER BONAVENTURE (2015), Computer Networking : Principles, Protocols and Practice

[18] Sheikh et.al Role of Pocket tracer in learning Computer Network" International Journal of Advance Research in Computer and 
communication Engineering (IJARCCE), volume 3, issue 5, May 2014.

[19] Sayed Mansoorhashimi, et.al "Performance Evaluation of Network Using Simulation Tools or Pocket Tracer", International organization of scientific Research (IOSR) of computer Engineering, volume19, issue 1,Jan.- Feb. 2017. https://doi.org/10.9790/0661-1901010105

[20] Edward E. et.al Modeling Network Router, Switches, And Security Using Cisco And OPNET Simulation Software" International organization of scientific Research (IOSR) of computer Engineering, volume 4, issue 7, July 2014.

https://doi.org/10.9790/3021-04734450

[21] S.V.R.K.Rao, et.al "Wireless sensor Network based Industrial Automation using Internet of Things(IOT)"'International Journal Of Advanced Trends In Computer Science And Engineering, Vol.7, No.6, Pages : 82-86, (2018). https://doi.org/10.30534/ijatcse/2018/01762018

[22] Sang Boem Lim, "Classification and Big Data Usages for Industrial Applications" International Journal of Advanced Trends in Computer Science and Engineering, 8(4), July- August 2019, 1117 1122 .

https://doi.org/10.30534/ijatcse/2019/18842019 